\title{
Experimental Research in Urban Spatial Cognition by Using Virtual Reality Technology
}

\author{
By Sinan Yuan* \\ Shuang Song ${ }^{\dagger}$ \\ Yukun Zhang ${ }^{*}$
}

This paper presents a VR experiment investigating how people construct the cognitive map in mind, generate spatial memory and respond when exploring the urban environment. 76 participants were recruited to explore the virtual town of Xidi. By analyzing the traces of the participants and the data of subjective feedbacks, the research examines how the space affects people generating spatial memory and producing the corresponding behavior pattern during the exploration. Furthermore, the interaction mechanism between the urban evolution and human behavior is investigated by comparing the participants' behavior trajectory and the real town spatial structure. Results from this research shed light on the future use of VR technology as an efficient tool in cognitive urban research. ${ }^{l}$

\section{Introduction}

The development of three-dimensional (3-D) computer graphics and related technology in recent years has led to a blurring of the distinction between reality and virtual environment. This suggests the validity of virtual reality (VR) technology as a tool for urban research. VR technology, directly associated with the human sensory organs, provides urban researchers new opportunities to investigate spatial cognition, memory and behavior in urban environment. It is advanced compared with animation cognition in the sense of providing the interactivity between people and environment. It involves the subjective judgment in spatial experience and simulates the entire process of cognition in real world. By using VR technology, it is possible to examine enormous objects such as urban space experimentally and study complicated phenomena analytically. Moreover, VR experiment makes it possible to study urban space among a wider population in comparison with conventional

\footnotetext{
*Lecturer, Tianjin University, China.

${ }^{\dagger}$ Architect, CCDI GROUP, China.

\$ Professor, Tianjin University, China.

${ }^{1}$ The paper is funded by the National Natural Science Foundation of China with the Foundation No: nsfc.51208346.
} 
methods. However, in the field of architecture VR technology is primarily used as a high performance media such as demonstration systems and aided design systems currently. Its application in the research of spatial cognition has seldom been explored.

This paper aims to expand the application of VR technology in urban science as an efficient tool in cognitive research. The paper presents a VR experimental method to investigate how urban morphological features affect people's spatial cognition and how people construct the cognitive map in mind, generate spatial memory and respond when exploring the urban environment.

The method was applied in Xidi, a typical Chinese settlement. The experiment addresses the following questions: 1 . how do people define the morphological characteristics of Xidi? 2. Whether and how does the space influence participants to produce corresponding behavior patterns during the exploration? 3. What factors are related to participants' choices at street junctions? 4 . What factors do people depend on to construct spatial memory and cognitive map?

\section{Spatial Cognition}

According to Bill Hillier(2005, p100), 'space is not a background to human activity, but an intrinsic aspect of everything human beings do in the sense that moving through space, interacting with other people in space, or even just seeing ambient space from a point in it.' All these behaviors are produced based on spatial cognition.

People's spatial cognition of street network is made up of an integration of cognitive memory fragments and the translation between them during the space exploration. It is an information processing procedure of the size, shape, orientation and distance of three-dimensional objects in physical space. It is concerned with the acquisition, organization, utilization, and revision of knowledge about spatial environments. These capabilities enable humans to manage basic and high-level cognitive tasks in everyday life.

The process of spatial cognition has three main phases: (1) obtaining the information of the surrounding environment; (2) capturing the morphological characteristics of space through various sensory organs; (3) storing the acquired information in brain, generating the understanding of the environment and constructing a cognitive map. The third phase plays the most important role in the whole cognition process. The term cognitive map was introduced by Tolman (1948), which later became the basis for cognitive psychology research. According to Kulpers (1983), the cognitive map is the body of knowledge of a large-scale environment which is acquired by integrating observations over time. It can be used to find routes and determine the relative position of places. Downs and Stea (2005, p314) offered cognitive mapping a formal definition as 'a process composed of a series of psychological transformations by which an individual acquires, codes, stores, recalls and decodes information about the relative locations and attributes of phenomena in his everyday spatial environment.' 
After 1950s, the research method of cognitive psychology was introduced in architecture and urban science. Lynch (1960) investigated spatial cognition through interviews, questionnaires and cognitive mapping. The investigation of people's cognitive mechanism of urban space has contributed to improving the quality of urban space and creating people-oriented settlement environment. In recent years, some researchers have begun to apply new methods such as VR technology in the investigation of spatial cognition. Wiener et al. (2009) explored how the geometry of space controls visual attention during spatial decision making by using VR technology. Frankenstein et al. (2010) used VR simulation to demonstrate how geometry and objects influence local route choices during way finding.

\section{Methods}

We build up a VR experiment platform as a cognitive research device. By integrating quantitative morphological research methods, the research investigates the relationship between people's behavior, cognition and environment.

\section{Space Syntax}

Space syntax, a popular quantitative research method of network analysis, was proposed by Bill Hiller et al. based on spatial cognition. The theory focuses on the topological structures of urban space. By analyzing the space configuration, it reveals intrinsic features of urban and architectural environment.

Axial map and visibility graph analysis (VGA) are two primary tools of space syntax for the analysis and representation of urban spatial structure. They can be performed by the computer program Depthmap developed by UCL.

'An axial map of the open space structure of the settlement is the least set of axial lines which pass through each convex space and make all axial links' (Hillier \&Hanson, 1984, p91-92).After translating an urban layout into an axial map, the map can be used to analyze the integration pattern of the street network. In an axial map, integration values of different streets are visualized by a color range (from red for the most integrated to blue for the least). The values also suggest the accessibility of topological lines for the entire street system. According to Penn (2003), an axial map represents the set of most efficient potential paths through an environment with respect to the accruing of maximum visual information about that context. It has been used successfully to investigate the effect of space structure on pedestrian movement (Hillier et al, 1987, 1993; Peponis et al, 1989). It is also effective in predicting navigational patterns (Penn, 2003).

VGA is used to analyze the variety of the integration inside a visual field. A uniform grid of points is generated within the entire area to be analyzed. By calculating the visible field from each point to any others, the grid is colored up according to the integration values from red (high) to blue (low). The resulting 
pattern of visual integration says more than how much you can see from each point. It also says how difficult it is to see all the space in the layout from each point. In the early days, VGA was used to analyze the inter-visibility connections within buildings and urban networks (e.g., Turner et al, 2001; Desyllas \&Duxbury, 2001). Recent researches (Proceedings of the Seventh International Space Syntax Symposium, 2009) show that VGA has been primarily applied to investigate how the structure of urban space has an effect on spatial accessibility and centrality under the guidance of vision. VGA can also be used to analyze the land use characteristics and pedestrian movement under the influence of spatial cognition.

\section{VR Experiment Platform}

The proposed VR experiment platform is designed to construct characteristics of virtual urban environment for experience, trace the participants and perform the research of spatial cognition.

A complete set of the platform is composed of a software system and a hardware system. The software system is used to simulate scenes of environment and achieve the real-time interactive behavior between people and environment. The hardware system outputs the information of scenes to participants for creating an immersive virtual environment and providing a man-computer interaction control interface (see Table1 and Fig.1).

Quest-3D which is developed by Act-3D is chosen as the core of the software workflow. By importing Sketch up or Autodesk 3ds Max models, Quest-3D will produce a real-time 3D virtual environment.

Table 1. The Component of VR Platform

\begin{tabular}{|l|l|l|}
\hline Subsystem & Functional unit & Uses and working principle \\
\hline \multirow{5}{*}{$\begin{array}{l}\text { Software } \\
\text { system }\end{array}$} & $\begin{array}{l}\text { Sketch up } \\
\text { Autodesk 3ds Max }\end{array}$ & $\begin{array}{l}\text { Modeling software } \\
\text { Generate .x model files }\end{array}$ \\
\cline { 2 - 4 } & Quest-3D & $\begin{array}{l}\text { Import .x model files, conduct the VR } \\
\text { environment settings (such as sunshine, sky, } \\
\text { control mode, behavior pattern, collision } \\
\text { detection, etc.), export the VR images and track } \\
\text { participants' movement. }\end{array}$ \\
\hline \multirow{5}{*}{$\begin{array}{l}\text { Hardware } \\
\text { system }\end{array}$} & Graphic workstation & $\begin{array}{l}\text { Construct and run VR scenes. Graphic } \\
\text { workstation with Quadro graphic card. Stereo } \\
\text { signal output and synchronizing function with 3D } \\
\text { glasses }\end{array}$ \\
\cline { 2 - 4 } & Display device & $\begin{array}{l}\text { 3rojecting beamers. Frame sequential/top } \\
\text { bottom 3D sync function }\end{array}$ \\
\cline { 2 - 4 } & 3D glasses & $\begin{array}{l}\text { LCD shutter glasses. Acquire stereoscopic vision } \\
\text { Achieve the interactive action between } \\
\text { participants and VR scenes. Mouse, keyboard, } \\
\text { gamepad, joystick and gloves etc. can be used. }\end{array}$ \\
\hline
\end{tabular}


Figure 1. Hardware System Workflow of VR Experiment Platform

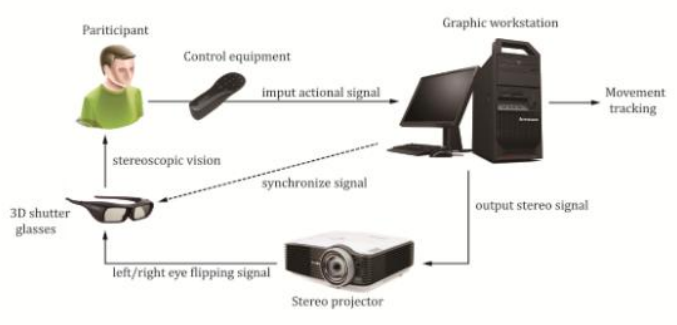

For the hardware system, a professional graphic workstation is used to connect projectors for image playback and interactive control. The virtual environment is projected through 3 projecting beamers on an ultra-wide screen at a resolution of $3840 \times 960$ pixels (see Fig.2). The screen refresh rate is $60 \mathrm{~Hz}$. The hardware system also includes 3D glasses for providing a full stereoscopic vision and control equipments for interactivity.

Quest-3D is also used to track and record the movements of participants. The spatial position data and time sequences of participants can be exported to Arc GIS for visualization. This achieves the virtual GPS tracking research in VR environment and the investigation of the overall behavior trend of largescale population in a particular space under the influence of the physical environment.

Figure 2. 3 Projecting Beamers

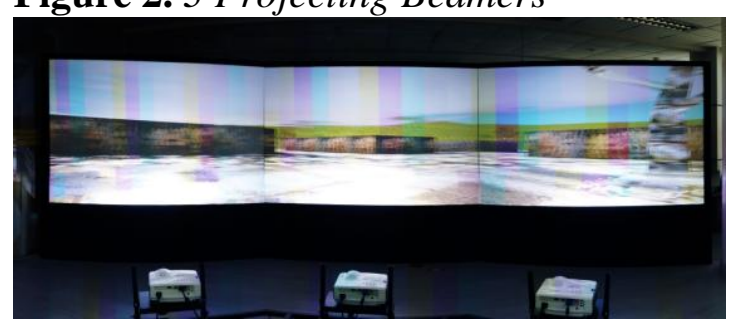

\section{Virtual Environment Modeling}

In a real environment, multiple factors act on people in their exploration at the same time. So it is hard to perform the analytic research on specific factors. In this research, a virtual town is modeled only retaining its spatial cues to insure that non-spatial cues such as road signs, crowd activities or plants would not affect explorers (see Fig.3). 
Figure 3. Modeling of the Virtual Xidi

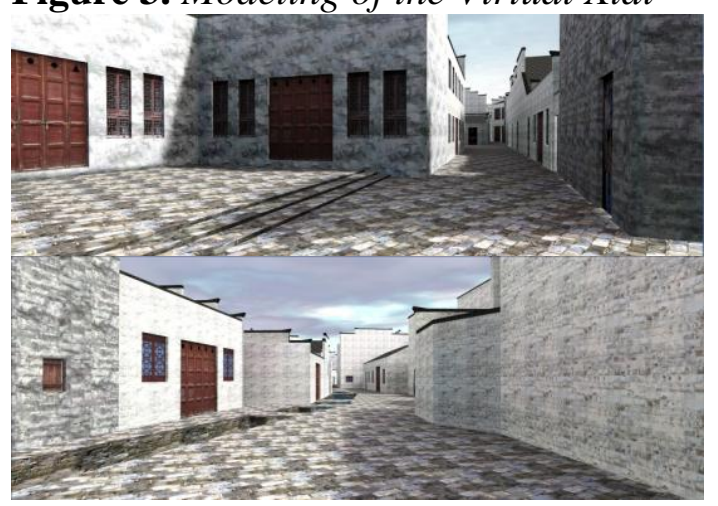

\section{Participants}

Participants are recruited to experience the virtual environment. The duration of each exploration is controlled in approximately 15 minutes to make sure that participants can fully experience the town street space and retain an effective memory of the street network after the exploration.

\section{Questionnaire}

Questionnaires are used to obtain participants' spatial cognition of the spatial morphological features though multiple-choice questions. The questions are set brief and direct to avoid hinting and misleading to participants. The participants are asked to redraw the street structure according to their memory. This implies the way they construct the spatial memory and cognitive map.

\section{Procedure}

The research recruits participants to experience the virtual street space. Participants are asked to wear 3D glasses and explore the virtual town by using control equipment (See Fig.4). Because there is no designated destination, participants could make free path choice in the street network and continuously update the exploration. They are told to try their best to remember the street network. Participants' movement is tracked and recorded in real-time mode during the exploration. After the VR experience, participants fill out the questionnaires and redraw the street structure.

Figure 4. VR Experiment

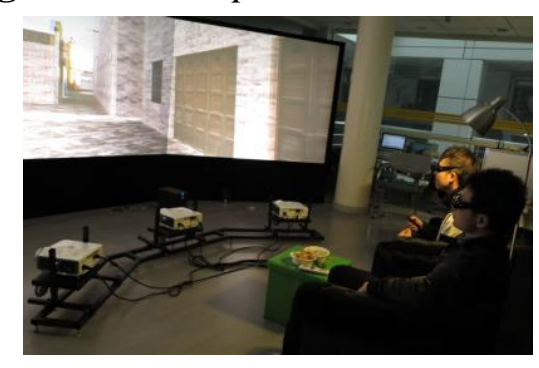




\section{Empirical Research of Xidi}

Xidi, a typical Chinese natural settlement, is selected as a case study using the proposed VR experiment platform. Located in the south of Anhui Province of China, Xidi was listed as a World Cultural Heritage Site in 2000. It preserves to a remarkable extent the appearance of ancient settlements of a type that largely disappeared or was transformed during the last century. This suggests its significance for the morphological research of Oriental settlements.

The formation and evolution of Xidi town structure is a self-organization growth process. It started from several scattered messuages on the eastern upland. After 1450, the town grew westward along the Front Stream, corresponding to the traditional Chinese concept "living by water". The town center shifted to the west after the construction of the ancestral hall (named as Hall of Respect). Then the town began to expand in north-south direction with the growth of population. The center of Xidi moved to the north while the Dalu Street became a primary street. The main entrance of the town locates at the lower reach of the stream and the west end of Dalu Street. It is the only formal access point for visitors. A ritual plaza was also formed at this place (Duan, 2006). With the passage of time, the town developed gradually and finally generated a stable resultant form which is about 700 meters from east to west and 300 meters from north to south. This progress explains the boatshape, irregularity, and curve which characterize the street network of Xidi to this day.

\section{Morphological Features of Xidi Street Network}

According to the functions, scales and the degrees of public character, the streets in Xidi can be categorized into three types: primary streets, local streets and access roads (See Fig.5). The primary streets including Dalu Street, Frontstream Street and Zhilu Street have the function of circulation distribution at the town scale. The social activities congregate in open spaces in front of the ancestral halls along the primary streets. These streets also function as commercial places in the town. Local streets mainly distribute the local circulation, connect residential groups and have mixed life uses. Access roads are mostly the alleys between buildings and provide frontage accesses. Most the streets in Xidi are curved except the straight and narrow alleys on both sides of ancestral halls. T-junctions are the majority in the street network of Xidi. 
Figure 5. Street Types of Xidi

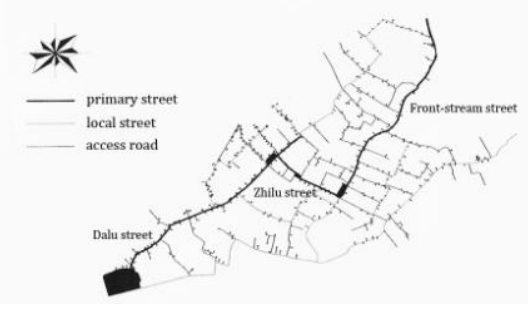

Figure.6 illustrates the space syntax analysis of Xidi street network by using Depthmap. The axial analysis (Fig. 6(a)) reveals the pattern of the topological integration of Xidi network. The red axial lines in the core of the street network are the connections among Dalu Street, Front-stream Street and Zhilu Street. The axial map represents an explicit topological centrality. The level of the integration descends from the core of the street network to the edge. Figure.6 (b) illustrates the VGA of Xidi network. From the macroscopic perspective, three primary streets have relatively high visual integration in VGA which exhibits similar spatial integration features with the axial map. But it reveals the microcosmic variety in the space of streets.

Figure 6. (a) analyzed axial map of Xidi; (b) VGA of Xidi

(a) (b)

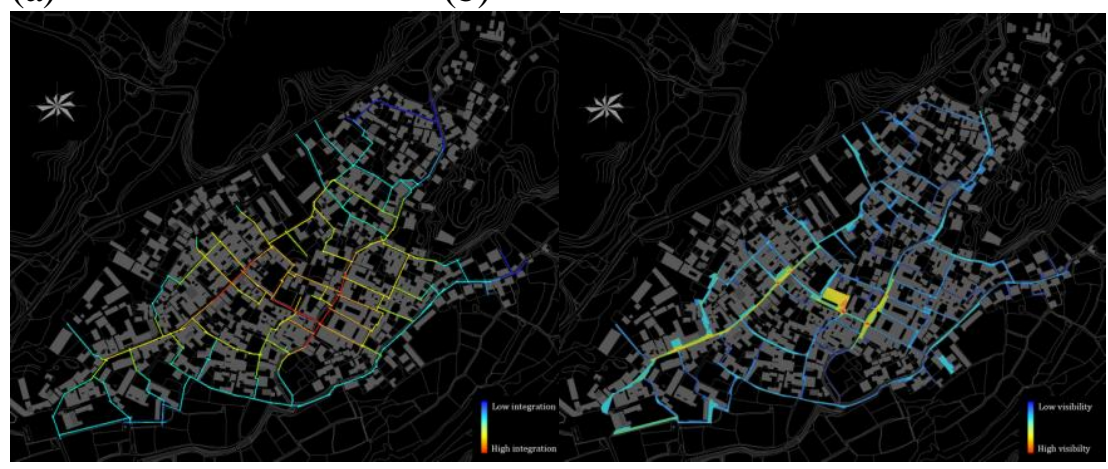

Experiment and Findings

The VR experiment is applied in the Xidi research. 76 participants experienced the virtual town and filled out the questionnaires.

\section{Movement Tracking}

The movement pattern of the participants presents the behavior logic during the spatial exploration, and further reveals the intrinsic cognitive mechanisms.

The starting point for each participant is set at this entrance to simulate the urban space exploration from the perspective of visitors. 54 effective traces of participants were recorded during the experiment. We imported these traces into ArcGIS and made a density analysis to visualize the frequency of passing though. The pattern of participants' movement during the exploration is shown 
in Figure 7. The color scale is a spectral range where red indicates the highest frequency down to blue for the lowest one. The movement pattern reveals a main spatial structure of the town to some extent.

\section{Figure 7. Intensity of Participants' Movement}

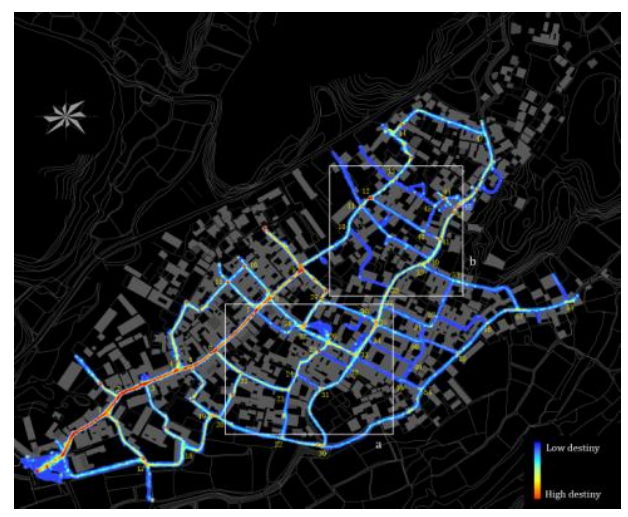

The main stream of people, which is shown as the red areas of the movement intensity diagram, mostly distributes on Dalu Street. Front-stream Street and some branches of Dalu Street close to the entrance are shown in yellow which indicates less movement intensity than Dalu Street. The local streets and access roads are less visited by participants and shown in blue. Although the experiment only retains the spatial cues and eliminates the effect of the commerce, it presents a similar pattern with the commercial distribution in the real town. The result of tracking analysis proves that the movement of people is not only driven by urban functional factors but also by space.

Based on this, the research further investigates how the spatial morphology of the town influences participants to produce corresponding behavior patterns during the exploration. The comparison between the movement intensity analysis (see Fig.7) and the axial map analysis of Xidi (see Fig.6 (a)) shows a similarity between the topological structure and the movement pattern. Most of the streets with high integration are those with high movement intensity such as Dalu Street and Front-stream Street, while the streets with low integration are rarely explored by participants. This verifies the impact of topological structure on spatial cognition and behavior, which is suggested by the Space Syntax theory. But this accordance is not always true in some local areas. For instance, some branches of Front-stream Street do not accord with the prediction of the analyzed axial map (see Fig.8). This could be explained by comparing the movement intensity and the VGA. 
Figure 8. Comparison between the Axial Map and the Movement Intensity

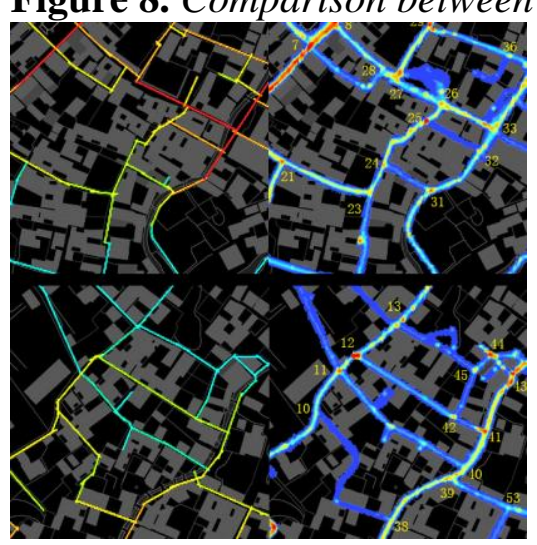

Figure 9 illustrates the comparison between the movement intensity and the VGA at all the network junctions. It helps to examine the effect of the morphological features of junctions on participants' behavior. At the junctions, the directions which participants preferred to choose usually have higher visual integration. Meanwhile, some junctions show equal visual integration in each direction that generates even movement intensity. We extracted the junctions where the axial map is different from participates' distribution (see Fig.10). Although some branch streets are high integrated in topological structure, lack of visibility at the corner makes people take the path in other direction.

Figure 9. Comparison of the Movement Intensity and the VGA at all Junctions

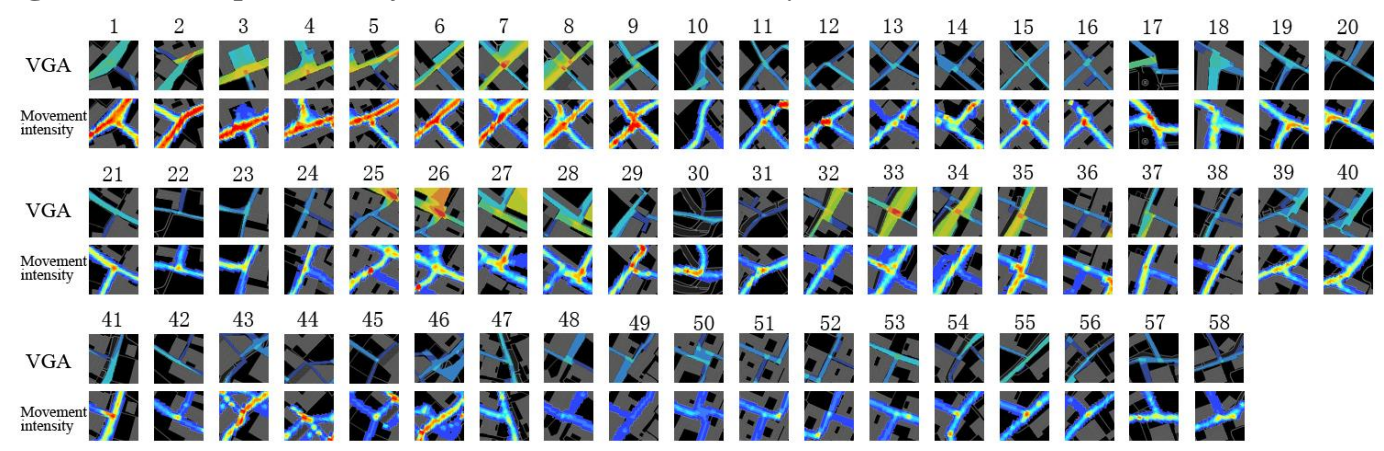

Figure 10. Comparison of the Movement Intensity and the VGA at Specific Junctions

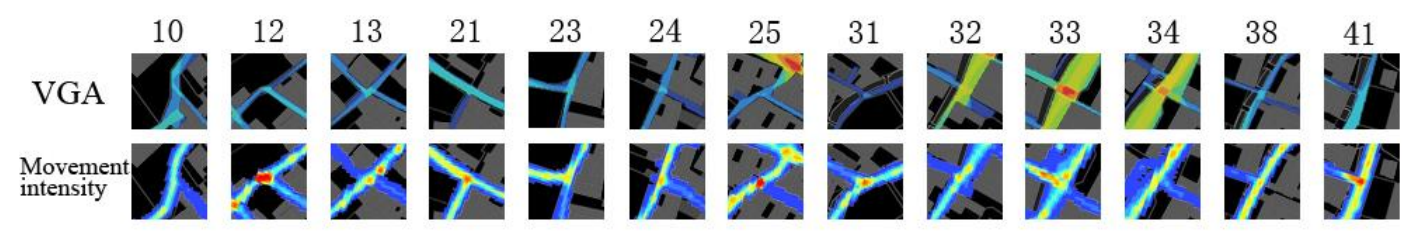

The results of the analysis indicate the fact that the spatial movement is impacted by topological structure and space form together in different scales.

Questionnaires

The questionnaire is as following: 
Please select the description which matches your feelings best of the virtual street space.

1. The width of the virtual town streets you feel is

A. extremely wide B. relatively wide C. normal D. relatively narrow E. extremely narrow

2. Walking in the virtual town you feel

A. extremely easy to find the direction B. relatively easy to find the direction

C. normal D. relatively easy to get lost E. extremely easy to get lost

3. You feel the virtual streets are

A. extremely curve B. relatively curve C. normal D. relatively straight E. extremely straight

4. You feel the virtual streets are

A. extremely characteristic B. relatively characteristic C. normal D. relatively common E. extremely common

5. Your overall impression of the virtual town is

A. interesting B. normal C. boring

A five point scale containing two extremes and a neutral option is set for each of the first four questions. Participants select the one which matches their spatial cognition best from the five options of each question.

Eventually, we collected 76 effective feedback questionnaires. The statistical analysis of them is shown in Figure 11. For each question, there is one option selected much more frequently than others. The second and third most selected options are always next to the most selected one. This indicates that people have an explicit judgment on spatial morphology in VR environment.

Figure 11. Questionnaire Option Statistics

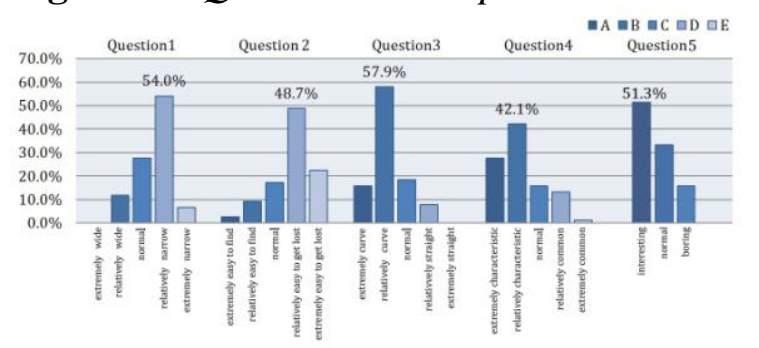

The majority of participants $(54 \%)$ considered the width of the virtual town streets was relatively narrow. Many participants $(48.7 \%)$ found it relatively easy to get lost when they were walking in the virtual town. A large proportion of participants (57.9\%) felt the virtual streets were relatively curve. $42.1 \%$ of the participants considered the virtual town streets relatively characteristic. The majority of the participants (51.3\%) considered the virtual town was interesting. 


\section{Cognitive Map}

The research collected 46 cognitive maps. Resemblances are shown in participants' drawings even though all the drawings are different from each other and also from the real map (see Fig.12). The factors which influence participants to generate the spatial memory and construct the cognitive maps in mind are identified by analyzing the drawing maps.

Figure 12. Cognitive Maps
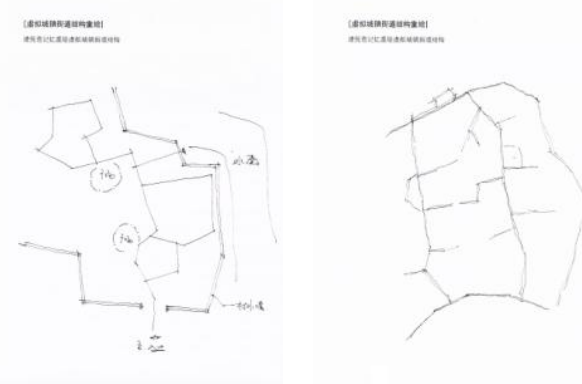

The factors are classified into three types including junctions, turns and objects of reference. Junctions were used most often in 22 drawing maps. Half of the drawings referenced to junctions, imagining a map of the environment where the path is visualized. Objects of reference such as buildings, squares, rivers and trees helped participants to reconstruct the itineraries in 20 maps. The sequences of turns were drawn to describe the exploration in 17 maps. Some participants depended on two factors at the same time to remember the street network.

\section{Conclusions}

There is a similar pattern to a great extent among the overall spatial structure, local morphology of space, people's movement, the functional structure of the real town and the street typology. This exhibits the systematic integrity of the self-organization town. In the urban evolution process, all the factors interact with and improve each other: the spatial morphology has effects on people's behavior; the later influences the urban functional structure; the functional structure further impacts the development and the internal reconstruction of space. This is a slow process in which good factors are retained while inappropriate ones are eliminated. Finally an ideal system is formed.

The experiment results show that the self-organization town has a coherent and explicit characteristic. The participants generated similar spatial cognition even though they experienced the streets of the virtual town in different regions and sequences. Although without a systematic plan, the town still maintains the continuity of the overall morphological characteristic during the growth, avoiding the problem of spatial fragmentation in modern city. Meanwhile, the overall characteristic is rich in variety rather than dull, in accordance with the 
questionnaires that $51.3 \%$ of the participants considered the experience were interesting.

Furthermore, the research also presents an experimental method of VR to investigate people's spatial cognition and behavior. Comparative analysis between the street morphological structure and people's movement is applied for the research of Xidi. The results show that the space of the street network has a significant correlation with people's behavior pattern. The results also provide a clue about how people construct a cognitive map in spatial experience. Junctions, turns and objects of reference are the key factors during the procedure of cognitive map construction.

To sum up, this experimental research put light on the future use of VR technology as an efficient tool in cognitive urban research. As suggested by the experiment, VR technology can play a key role in the research of spatial cognition, memory and behavior in different environment.

\section{Bibliography}

Colom, R., Contrera, M. J., Botella, J. \& Santacreu, J. (2001). 'Vehicles of spatial ability'. Personality Individual Differences 32: 903-912.

Desyllas, J. \&Duxbury, E. ( 2001). Axial Maps and Visibility Graph Analysis. A comparison of their methodology and use in models of urban pedestrian movement. Proceedings of the 3rd International Space Syntax Symposium, May, in Atlanta.

Downs, R. \& Stea, D. (2005) Images and environment: cognitive mapping and spatial behavior. Chicago: Transaction Publishers.

Duan, J., Gong, K., Chen, X.D., Zhang, X.D \& Peng, S. (2006).Urban space 1.The spatial analysis of the traditional town Xidi, a World Cultural Heritage Site. mechanisms in China. Nanjin: Southeast University Press. [In Chinese].

Frankenstein, J., Buchner, S.J., Tenbrink, T. \&Holsher, C. (2010). 'Influence of geometry and objects on local route choices during wayfinding'. Spatial cognition VII: 41-53.

Hillier, B. (2005). 'The art of place and the science of space'. World Architecture 2005 (11):96-102.

Hillier, B. \& Hanson J. (1984). The Social Logic of Space. Cambridge: Cambridge University Press.

Hillier, B., Burdett, R., Peponis, J. \& Penn, A. (1987). 'Creating life: or, does architecture determine anything? ' Architecture and Behavior 3: 233 -250.

Hillier, B., Penn, A., Hanson, J., Grajewski, T \& Xu, J. (1993). 'Natural movement: or, configuration and attraction in urban pedestrian movement'. Environment and Planning B: Planning and Design 20: 29-66.

Kulpers, B.J. (1983). 'The cognitive map: could it have been any other way?' In: Spatial Orientation, 345-359. New York: Plenum Press.

Lynch, K. (1960). The image of the city. Cambridge: MIT Press.

Penn, A. (2003). 'Space syntax and spatial cognition: Or why the axial line?' Environment \&Behavior, 35, 30-65.

Peponis, J., Hadjinikolaov, E., Livieratos, C \& Fatouros, D.A. (1989). 'The spatial core of urban culture'. Ekistics 56(334/335): 43 -55. 
Vol. 1, No. $1 \quad$ Yuan et al: Experimental Research in Urban Spatial Cognition ...

Royal Institute of Technology. (2009). Proceedings of the Seventh International Space Syntax Symposium.

Tolman, E.C. (1948). 'Cognitive maps in rats and men'. Psychological Review 55 (4): $189-208$.

Turner, A., Doxa, M., O'Sullivan, D. \&Penn, A. (2001). 'From Isovists to Visibility Graphs: A Methodology for the Analysis of Architectural Space'. Environmental and Planning B: Planning\& Design 28(1):103-121.

Wiener, J.M., Holscher, C., Buchner, S.J. \& Konieczny, L. (2009). 'How the Geometry of Space controls Visual Attention during Spatial Decision Making'. Paper presented at the Annual Meeting of the Cognitive Science Society 2009, in Amsterdam, Netherlands. 